\title{
Ultrasonic scalpel with knot tying protects parathyroid function for total thyroidectomy with central neck dissection
}

\author{
Jun Jiang, Meiping Shen, Hui Lu \\ Department of General Surgery, The First Affiliated Hospital of Nanjing Medical University, Nanjing 210029, China \\ Contributions: (I) Conception and design: H Lu, M Shen; (II) Administrative support: H Lu; (III) Provision of study materials or patients: J Jiang; (IV) \\ Collection and assembly of data: J Jiang; (V) Data analysis and interpretation: J Jiang; (VI) Manuscript writing: All authors; (VII) Final approval of \\ manuscript: All authors. \\ Correspondence to: Hui Lu; Meiping Shen. Department of General Surgery, The First Affiliated Hospital of Nanjing Medical University, No. 300 \\ Guangzhou Road, Nanjing 210029, China. Email: luhui@jsph.org.cn; shenmeiping@jsph.org.cn.
}

\begin{abstract}
Backgroundk Hypoparathyroidism might be due to collateral thermal injury induced by harmonic scalpel (HS) after total thyroidectomy (TT) with central neck dissection (CND). The current study aimed to evaluate whether a clamp and tie (CAT) technique used to preserve parathyroid glands in situ could reduce damage to parathyroid function in HS patients undergoing TT plus unilateral or bilateral CND.

Methods: Medical records of 537 HS-operated patients with papillary thyroid carcinoma (PTC) undergoing TT plus unilateral or bilateral CND were retrospectively evaluated. Patients were divided into HS and HS-CAT groups based on whether the CAT techniques were used near parathyroid glands. Patients' characteristics such as age, sex, tumour size, operative time, pre- and post-operative levels of parathyroid hormone (PTH) and complications were analysed.

Results: For patients undergoing TT plus unilateral CND, operative time was significantly shorter $(\mathrm{P}<0.001)$, but there were significantly higher incidences of transient hypoparathyroidism $(\mathrm{P}=0.002)$ on postoperative day 1 and incidental parathyroidectomy $(\mathrm{P}=0.036)$ in the HS group. There was no significant difference in permanent hypoparathyroidism. For patients undergoing TT plus bilateral CND, a significantly shorter operative time and sharper postoperative PTH decline was found in the HS group $(\mathrm{P}=0.029)$. However, there were no significant differences regarding incidences of incidental parathyroidectomy, transient or permanent hypoparathyroidism.
\end{abstract}

Conclusions: The CAT technique applied near parathyroid glands was a practical surgical application for decreasing the incidence of postoperative transient hypoparathyroidism for TT plus unilateral CND.

Keywords: Cold knife; knot tying; harmonic scalpel (HS); hypoparathyroidism; total thyroidectomy (TT)

Submitted Sep 21, 2019. Accepted for publication Jan 04, 2020.

doi: 10.21037 /gs.2020.01.12

View this article at: http://dx.doi.org/10.21037/gs.2020.01.12

\section{Introduction}

Hypoparathyroidism following total thyroidectomy (TT) is a common complication. The reported incidence of hypoparathyroidism is variable depending on the surgical extent and the definition of this complication (1-3). Hypoparathyroidism may result from direct mechanical injury, devascularization, thermal trauma, oedema, or inadvertent removal of the parathyroid during surgery, which are also associated with the extent of thyroidectomy, the surgical techniques and surgeon's experience (4-6).

Currently, Ethicon's Harmonic Scalpel (HS) FOCUS Shears are widely used in thyroidectomy. The ultrasonically activated shears can safely dissect, coagulate and simultaneously cut tissue, thus reducing the operative time $(7,8)$. Although the lower energy and temperature of HS can produce less thermal damage to the surrounding important structures, water boiling and vapour around the 
tissue coagulated by HS can still occur. Considering the characteristics of the fragile blood supply of parathyroid glands (PTGs) $(9,10)$, it is reasonable to speculate that using a conventional clamp and tie (CAT) technique near PTGs other than HS may help to protect parathyroid function and reduce the risk of postoperative hypoparathyroidism (11).

This study evaluated the impact of two surgical techniques (HS or CAT) to isolate and preserve the PTGs near PTGs on the incidence of transient and permanent hypoparathyroidism in patients undergoing TT with unilateral or bilateral central neck dissection (CND).

\section{Methods}

A retrospective review of patients with papillary thyroid carcinoma (PTC) who underwent TT with unilateral or bilateral CND between $1^{\text {st }}$ October 2017 and $31^{\text {st }}$ December 2018 was conducted. Exclusion criteria were preoperative need for calcium or vitamin D supplementation, preoperative hypocalcaemia, concomitant parathyroid disease, not diagnosed PTC, and a previous thyroidectomy. Patients with incomplete follow-up information were also excluded. All operations were performed by an experienced surgeon $(\mathrm{H} \mathrm{Lu})$. The study protocol was approved by the Ethics Committee of the first affiliated hospital of Nanjing medical university (number of ethical approval: 2018-SR209). Informed consent was not required since this was a retrospective study and all data with personal identifiers were kept confidential.

\section{Surgical methods}

Included patients were divided into the HS and HS-CAT groups. All operations were performed using HS in the HS group of patients admitted between $1^{\text {st }}$ October 2017 and $31^{\text {st }}$ May 2018. In the HS-CAT group of patients admitted between $1^{\text {st }}$ June 2018 and $31^{\text {st }}$ December 2018, all operations were performed using HS except that isolation and preservation of the PTGs were achieved by the traditional CAT technique using 4-0 Vicryl suture (Ethicon, Inc.).

The decision of TT and the extent of CND (unilateral or bilateral) were performed based on the American Thyroid Association guidelines (12), patients' willingness and surgeon's experience. Prophylactic CND was routinely performed based on surgeon's experience and skills, with TT and CND performed as previously described $(12,13)$.
In addition, CND was performed after TT. Specifically, the thyrothymic ligament was exposed first to observe the inferior parathyroid along this ligament at the beginning of the operation. During the operation, the superior and inferior PTGs were not routinely completely exposed but these glands as well as the adipose tissue around them were maintained in situ. At the beginning of CND procedure, the thyrothymic ligament and the inferior PTGs were retracted laterally as previously reported. Incidentally, excised PTGs found before frozen sections were autotransplanted into the ipsilateral sternocleidomastoid muscle during the operation as previously reported (14).

\section{Clinical parameters}

Clinical parameters investigated included age, sex, maximum tumour size, gross extrathyroidal extension, bilaterality, multifocality, preoperative total serum calcium, perioperative parathyroid hormone (PTH) levels, PTH decline [calculated as (preoperative $\mathrm{PTH}$ - postoperative $\mathrm{PTH}) \times 100 /$ preoperative $\mathrm{PTH}$, the incidence of hypoparathyroidism, operative time, number of harvested lymph nodes, number of PTGs auto transplanted, number of PTGs found in the final pathology and other surgical complications.

\section{Definition of bypoparathyroidism}

The normal range of serum PTH assay at our hospital is between 12 and $88 \mathrm{pg} / \mathrm{mL}$. Postoperative hypoparathyroidism was defined as serum $\mathrm{PTH}$ value $<12 \mathrm{pg} / \mathrm{mL}$, regardless of the level of total serum calcium and the presence of hypocalcaemic symptoms on postoperative day 1 (POD 1) (15). Permanent hypoparathyroidism was defined as serum PTH level $<12$ $\mathrm{pg} / \mathrm{mL} 6$ months after surgery. Oral calcium and vitamin $\mathrm{D}$ were initiated based on PTH and total serum calcium measurements on POD 1, with calcium supplementation further individualised in follow-up. Patients were followed for at least 6 months.

\section{Statistical analysis}

Continuous data are presented as mean \pm standard deviation or the median with interquartile ranges. Clinical parameters were compared using Student's $t$-test or Mann-Whitney $\mathrm{U}$ test for continuous data and using the chi-square test or 
Table 1 Demographics and clinical characteristics of patients who underwent total thyroidectomy with unilateral central neck dissection

\begin{tabular}{|c|c|c|c|}
\hline Variable & $\mathrm{HS}(\mathrm{n}=238)$ & HS-CAT (n=209) & $\mathrm{P}$ \\
\hline Female (\%) & $166(69.7 \%)$ & $158(75.6 \%)$ & $0.167^{\ddagger}$ \\
\hline Tumor size $(\mathrm{cm})$ & $1.0(0.7-1.5)$ & $1.0(0.7-1.7)$ & $0.790^{\dagger}$ \\
\hline Gross extrathyroid extension (\%) & $32(13.4 \%)$ & $24(11.5 \%)$ & $0.532^{\ddagger}$ \\
\hline Multifocality (\%) & $79(33.2 \%)$ & $81(38.8 \%)$ & $0.221^{\ddagger}$ \\
\hline Hashimoto's thyroiditis (\%) & $54(22.7 \%)$ & $49(23.4 \%)$ & $0.850^{\ddagger}$ \\
\hline Preoperative PTH (pg/mL) & $45.85(34.9-56.9)$ & 42.7 (32.9-55.3) & $0.139^{\dagger}$ \\
\hline Preoperative serum calcium (mmol/L) & $2.3 \pm 0.1$ & $2.2 \pm 0.1$ & $<0.001^{\S}$ \\
\hline Hematoma (\%) & $6(2.5 \%)$ & $4(1.9 \%)$ & $0.756^{\pi}$ \\
\hline \multicolumn{4}{|l|}{ Vocal cord paralysis (\%) } \\
\hline Temporary & $4(1.7 \%)$ & $3(1.4 \%)$ & $1^{\pi}$ \\
\hline Permanent & $2(0.8 \%)$ & $1(0.5 \%)$ & $1^{\pi}$ \\
\hline Incidental removal of parathyroid (\%) & $26(10.9 \%)$ & $11(5.3 \%)$ & $0.030^{\ddagger}$ \\
\hline Found around the gland & $9(3.8 \%)$ & $3(1.4 \%)$ & $0.151^{\pi}$ \\
\hline Found in central compartment & $17(7.1 \%)$ & $8(3.8 \%)$ & $0.128^{\ddagger}$ \\
\hline Parathyroid autotransplanted (\%) & $30(12.6 \%)$ & $14(6.7 \%)$ & $0.036^{\ddagger}$ \\
\hline
\end{tabular}

PTH decline was calculated as (preoperative PTH - postoperative PTH) $\times 100 /$ preoperative PTH. Continuous data are presented as mean \pm standard deviation or the median with or without interquartile ranges. ${ }^{\dagger}$, determined using Mann-Whitney $\mathrm{U}$ test; ${ }^{\ddagger}$, determined using Chisquare test; ${ }^{\S}$, determined using Student's $t$-test; " , determined using Fisher's exact test. HS, Harmonic Scalpel; CAT, clamp and tie; PTH, parathyroid hormone.

Fisher's exact test for categorical variables between the HS and HS-CAT groups (SPSS 13.0 for Windows). P values $<0.05$ were considered statistically significant.

\section{Results}

\section{Patients who underwent TT with unilateral CND}

A total of 447 patients who underwent TT with unilateral CND were divided into two groups: 238 in the HS group and 209 in the HS-CAT group. Patient characteristics are shown in Table 1. There were no significant differences between the HS and HS-CAT groups regarding patient age $(\mathrm{P}=0.100)$ and gender $(\mathrm{P}=0.167)$. Tumour characteristics including tumour size $(\mathrm{P}=0.790)$, extrathyroid extension $(\mathrm{P}=0.532)$, bilateral foci $(\mathrm{P}=0.416)$ and tumour multifocality $(\mathrm{P}=0.221)$ were also not significantly different between the two groups. The coexistence of Hashimoto's thyroiditis and PTC was similar for both groups $(\mathrm{P}=0.850)$.

There was no significant difference in preoperative $\mathrm{PTH}$ 
Table 2 Demographics and clinical characteristics of patients who underwent total

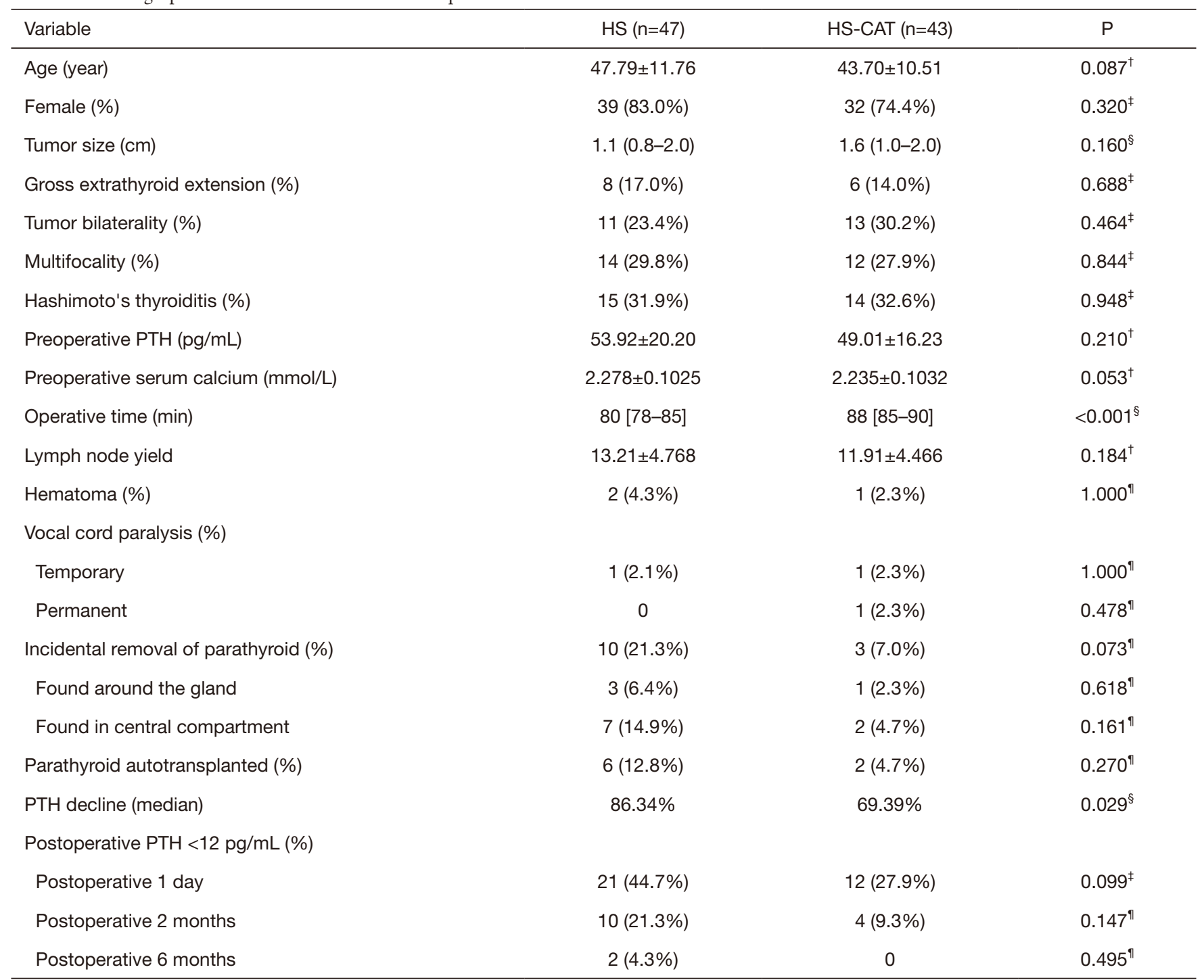

PTH decline was calculated as (preoperative PTH - postoperative PTH) $\times 100 /$ preoperative PTH. Continuous data are presented as mean \pm standard deviation or the median with or without interquartile ranges. ${ }^{\dagger}$, determined using Student's $t$ test; ${ }^{\ddagger}$, determined using Chi-square test; ${ }^{\S}$, determined using Mann-Whitney U test; " , determined using Fisher's exact test. HS, Harmonic Scalpel; CAT, clamp and tie; PTH, parathyroid hormone.

level between the HS and HS-CAT groups ( $\mathrm{P}=0.139)$. However, the HS group had a higher level of preoperative total serum calcium than the HS-CAT group $(\mathrm{P}<0.001)$. Furthermore, the duration of the operation was significantly longer for the HS-CAT group $(\mathrm{P}<0.001)$. There were no differences in the number of harvested central neck lymph nodes $(\mathrm{P}=0.080)$ and complication rates of hematoma $(\mathrm{P}=0.756)$, temporary $(\mathrm{P}=1.000)$ and permanent vocal cord paralysis $(\mathrm{P}=1.000)$.

The number of PTGs incidentally removed $(\mathrm{P}=0.030)$ and auto transplanted $(\mathrm{P}=0.036)$ was significantly higher for the HS group, but the median PTH decline was similar for both groups $(\mathrm{P}=0.219)$. The incidence of transient hypoparathyroidism on POD 1 was significantly higher in the HS than HS-CAT group (38.2\% vs. $24.4 \%, \mathrm{P}=0.002)$. The incidence of hypoparathyroidism on postoperative 2 months was higher in the HS group (9.7\%) than in the HS-CAT group (5.3\%), however this did not reach statistical significance $(\mathrm{P}=0.080)$. The incidence of permanent hypoparathyroidism was similar in both 
groups $(\mathrm{P}=0.626)$. The two patients with permanent hypoparathyroidism 6 months after surgery had not fully recovered from hypoparathyroidism at 1-year follow-up.

\section{Patients who underwent TT with bilateral CND}

A total of 90 patients undergoing TT with bilateral CND were divided into two groups: 47 in the HS group and 43 in the HS-CAT group. Patient baseline characteristics and surgical results are shown in Table 2. Patient age $(\mathrm{P}=0.087)$, gender $(\mathrm{P}=0.320)$, tumour size $(\mathrm{P}=0.160)$, extrathyroid extension $(\mathrm{P}=0.688)$, bilateral foci $(\mathrm{P}=0.464)$, multifocality $(\mathrm{P}=0.844)$ and the coexistence of Hashimoto's thyroiditis $(\mathrm{P}=0.948)$ were not significantly different between the two groups. There were also no significant differences in preoperative PTH level $(\mathrm{P}=0.210)$ and total serum calcium $(\mathrm{P}=0.053)$ between the HS and HS-CAT groups. However, the median operative time was significantly shorter for the HS group than for the HS-CAT group $(\mathrm{P}<0.001)$. The number of central neck lymph nodes was similar for the two groups ( $\mathrm{P}=0.184)$, as were surgical complications such as hematoma $(\mathrm{P}=1.000)$, temporary $(\mathrm{P}=1.000)$ and permanent vocal cord paralysis $(\mathrm{P}=0.478)$. Moreover, the two groups did not differ in terms of the number of PTGs incidentally removed $(\mathrm{P}=0.073)$ and auto transplanted $(\mathrm{P}=0.270)$. Nevertheless, if taking both unilateral and bilateral CNDoperated patients as a whole (537 patients), the incidence of incidentally removed and auto transplanted PTGs was both $12.6 \%(36 / 285)$ in the HS group (285 patients), and 5.6\% $(14 / 252)$ and $6.3 \%(16 / 252)$ in the HS-CAT group (252 patients), respectively. And correspondingly, there were significant difference with respect to the number of PTGs incidentally removed $(\mathrm{P}=0.005$, Chi-square test) and auto transplanted $(\mathrm{P}=0.014$, Chi-square test) between the HS and HS-CAT group.

The postoperative PTH level declined significantly in the HS group in comparison with the HS-CAT group $(86.34 \%$ vs. $69.39 \%, \mathrm{P}=0.029)$. The transient hypoparathyroidism rate in the HS group on POD 1 was much higher than in the HS-CAT group (44.7\% vs. $27.9 \%$, $\mathrm{P}=0.099)$, but this did not reach statistical significance. Similarly, though TT with bilateral CND using the CAT technique showed a prominent tendency to decrease transient hypoparathyroidism on postoperative 2 months, it was not statistical significantly different $(\mathrm{P}=0.147)$. There was no significant difference in the incidence of permanent hypoparathyroidism between the two groups $(\mathrm{P}=0.495)$. Of note, the parathyroid function of these two patients in the HS group was not fully recovered at 1-year follow-up.

\section{Discussion}

HS has been demonstrated to be a useful alternative to the conventional clamp and knot tying technique in performing thyroidectomy with comparable morbidity rates by many studies (16). However, other studies found that advantages of HS in reduction of postoperative complications were not statistically significant in comparison to the cold knife technique (17). It has been reported that the low temperature generated by HS will not cause the water within the tissues to boil, thus theoretically, HS induces little collateral thermal injury. However, in clinical practice, steam spread and even boiling water around the HS working rod can be observed, especially in some cases of thyroid oedema, such as Hashimoto's thyroiditis, or lymphatic leakage during dissection. It is not possible to fully exclude the possibility of HS induced collateral thermal injury to PTGs when exploring the underlying mechanisms of postoperative hypoparathyroidism after TT (11). Based on such concerns, we isolated and preserved PTGs using cold knife instead of energy based devices during TT since $1^{\text {st }}$ June 2018. In our centre, the use of different energy based devices or traditional techniques depend on surgeons' preferences. As the CAT technique is a traditional surgical skill and is not aggressive in comparison with ultrasonic knife, informed consent was not obtained from the patients when we decided to operate more carefully in the area near the PTGs with the CAT technique.

Here, we demonstrated that the practical tip of preserving the PTGs in situ with knot suture instead of HS in the vicinity of PTGs was helpful to reduce damage to the PTGs and decrease the incidence of transient hypoparathyroidism at the cost of prolonged operative time in patients who underwent TT plus CND. This result is quite different from previous findings, which may be due to many reasons. First, hybrid techniques including HS and knot tying were both used in the HS-CAT group in the current study, while previous studies focused the comparison between groups using a single technique, HS-CAT or HS alone in each group $(11,18-20)$. Second, the learning curve of HS and the extent of adherence to manufacturers' instructions for HS use might be different among thyroid surgeons in these studies. Inappropriate use of HS by inexperienced surgeons can result in potential 
damage to PTGs. Third, study subjects in the current study were all PTC patients, while other studies included patients with benign or malignant thyroid disease, or both. The mixed subjects underwent different surgical regimens including TT or unilateral thyroidectomy with or without CND or lateral cervical lymph node dissection in previous studies (2).

The protective role of the CAT technique in the current study may be due to more careful attention to identify and preserve PTGs in their orthotopic positions when applying a suture knot for haemostasis. It also took some time to make a knot tying. This may explain why more operative time was spent performing TT with unilateral CND in the HS-CAT group. Moreover, our data showed that the number of PTGs auto transplanted and incidentally excised was significantly decreased in the HS-CAT group in patients who underwent TT with unilateral CND. Our data showed that PTGs were incidentally removed more often during CND (Tables 1,2) in both the HS and HS-CAT groups. This is most likely because of the difficulty in distinguishing the PTGs from adipose tissue and lymph nodes. The suture knot might serve as a landmark of preserved PTGs, thus decreasing incidental parathyroidectomy during CND (21). Consistently, for patients who underwent TT with bilateral CND in the current study, the number of incidentally removed PTGs was also higher in the HS group than in the HS-CAT group, however without statistical significance, indicating that the CAT procedure potentially decreased the risk of incidental removal of PTGs. Another advantage of the hybrid technique of HS combined CAT in the HSCAT group is that the bloodless surgical field from the use of HS facilitates surgeons to better identify and preserve PTGs in situ. The decreased incidence of incidental parathyroidectomy may contribute to the protective effect of the CAT technique on parathyroid function. However, so far a link between inadvertent parathyroidectomy and postoperative hypoparathyroidism was not fully elucidated in the literature $(1,5,21,22)$. Based on our experience, loss of bulk or the whole relatively large parathyroid gland would immediately result in postoperative transient hypoparathyroidism. Sometimes, PTGs appear as a cluster of small parathyroid tissue and fragment resection of these small scattered PTGs might have little impact on postoperative hypoparathyroidism. This may explain why there were no differences with respect to the incidence of hypoparathyroidism on postoperative 2 months and permanent hypoparathyroidism between the HS-CAT and HS groups, though HS caused a relatively higher incidence of hypoparathyroidism in the current study. The existence of above-mentioned small scattered PTGs and some possible ectopic PTGs may also explain why incidental removal of the PTGs were found in the specimens of CND by the pathologist in both the HS and HS-CAT groups. As previously reported, deliberately identifying PTGs was conducive to preserve PTGs in situ (5). Other researchers found that visualising fewer PTGs contributed to the reduction in the incidence of hypoparathyroidism after TT (23). We believe that identifying and preserving the PTGs in situ with the CAT technique is critical to better preserve the blood supply to the PTGs undisturbed. Anatomic knowledge about vascular structures supplying PTGs is essential for surgeons to perform an individualised preservation regimen of the PTGs (10). Here, we did not routinely expose the parathyroid and identify the specific blood supply type not only because it was a time-consuming process, but also because it might damage the blood supply of PTGs (24). Therefore, we also did not routinely record the number of PTGs actually seen during the surgery. As previously reported and we observed $(4,9,10,25)$, some types of PTGs may be supplied by very subtle vascular branches from tertiary branches of inferior thyroid artery, or from many tiny anastomosing branches between the superior and inferior thyroid arteries at the posterior aspect of thyroid, or from vasa vasorum of the thyroid lobe or even from adipose tissue around PTGs. Of note, a small portion of PTGs receive their blood supply mostly from the thyroid lobe. It is nearly impossible to preserve a satisfactory blood supply of PTGs detached from the thyroid lobe in these situations $(25,26)$. Therefore, even meticulous capsular dissection with the CAT technique failed to completely avoid vascular damage to the PTGs and postoperative hypoparathyroidism for patients undergoing TT plus CND in the current study. It is worth noting that combination of the CAT technique with indocyanine green fluorescence (27) or carbon nanoparticles (28) may help to better preserve the blood supply by reducing unnecessary dissection of the surrounding tissue if the PTGS can be identified in advance as previously reported.

The protective effect of the CAT technique here may also be attributed to decreased collateral thermal spread damage of the HS working rod. When the PTGs are closely attached to the thyroid lobe, a safe $3 \mathrm{~mm}$ work distance for HS cannot be guaranteed, fragile blood vessels to PTGs may be interrupted by collateral thermal spread temporarily or permanently (26). The traditional capsular dissection with the CAT technique can better 
preserve these tiny lateral-to-medial vessels than HS, thus reducing the incidence of transient hypoparathyroidism. The adverse effects caused by thermal injury of HS, such as temporary vessel spasm or permanent protein denaturation, may be counteracted by vasodilatation, parathyroid cells regeneration and blood reperfusion from collateral blood supply over time.

Though the current study is a retrospective study, a power calculation was performed prior to the study to determine the required sample size to detect a true effect. Based on the incidence of hypoparathyroidism on postoperative 1 day collected earlier (35\% of the HS group vs. $21 \%$ of the HS-CAT group in the initial analysis), at least 161 patients with TT plus unilateral CND were required for each group, and at least 128 patients with TT plus bilateral CND (incidence of hypoparathyroidism: $38 \%$ of the HS group vs. $22 \%$ of the HS-CAT group in the initial analysis) were required for each group to achieve a power of $80 \%$ (26). However, in our centre, we tried to avoid performing bilateral prophylactic CND due to the increased possibility of complications, so there were relatively few patients undergoing TT plus bilateral CND, which might result in limited statistical power. For example, the postoperative PTH level of the HS group decreased more significantly than that of the HS-CAT group in patients who underwent TT plus bilateral CND, the incidence of hypoparathyroidism on postoperative 1 day and 2 months was not significantly different between the two groups. A too small sample size of patients who underwent TT with bilateral CND might also be a possible explanation for the lack of statistical significances in the incidence of incidentally removed and autotransplanted PTGs in this group. Moreover, our data showed that the HS group had a higher level of preoperative total serum calcium than the HS-CAT group in patients undergoing TT with unilateral CND. Whether the difference was attributed to sampling error was unknown. Whether a higher total serum calcium would influence calcium metabolism and preoperative PTH secretion in this group in the current study was unclear. Thus, our results should be carefully interpreted and further prospective research with rigorous statistical design is required.

\section{Conclusions}

The CAT technique is not new but a simple, cheap and practical surgical approach for reducing the short-term incidence of postoperative transient hypoparathyroidism, especially for patients following TT plus unilateral CND.

\section{Acknowledgments}

The authors thank Liping Zhang for her patience and support over the past years.

Funding: None.

\section{Footnote}

Conflicts of Interest: All authors have completed the ICMJE uniform disclosure form (available at http://dx.doi. org/10.21037/gs.2020.01.12). The authors have no conflicts of interest to declare.

Ethical Statement: The authors are accountable for all aspects of the work in ensuring that questions related to the accuracy or integrity of any part of the work are appropriately investigated and resolved. The study protocol was approved by the Ethics Committee of the first affiliated hospital of Nanjing medical university (number of ethical approval: 2018-SR-209). Informed consent was not required since this was a retrospective study and all data with personal identifiers were kept confidential.

Open Access Statement: This is an Open Access article distributed in accordance with the Creative Commons Attribution-NonCommercial-NoDerivs 4.0 International License (CC BY-NC-ND 4.0), which permits the noncommercial replication and distribution of the article with the strict proviso that no changes or edits are made and the original work is properly cited (including links to both the formal publication through the relevant DOI and the license). See: https://creativecommons.org/licenses/by-nc-nd/4.0/.

\section{References}

1. Su A, Wang B, Gong Y, et al. Risk factors of hypoparathyroidism following total thyroidectomy with central lymph node dissection. Medicine (Baltimore) 2017;96:e8162.

2. Chen KC, Iqbal U, Nguyen PA, et al. The impact of different surgical procedures on hypoparathyroidism after thyroidectomy: A population-based study. Medicine (Baltimore) 2017;96:e8245.

3. Mehanna HM, Jain A, Randeva H, et al. Postoperative hypocalcemia--the difference a definition makes. Head Neck 2010;32:279-83. 
4. Dedivitis RA, Aires FT, Cernea CR. Hypoparathyroidism after thyroidectomy: prevention, assessment and management. Curr Opin Otolaryngol Head Neck Surg 2017;25:142-6.

5. Paek SH, Lee YM, Min SY, et al. Risk factors of hypoparathyroidism following total thyroidectomy for thyroid cancer. World J Surg 2013;37:94-101.

6. Chadwick DR. Hypocalcaemia and permanent hypoparathyroidism after total/bilateral thyroidectomy in the BAETS Registry. Gland Surg 2017;6:S69-S74.

7. Revelli L, Damiani G, Bianchi CB, et al. Complications in thyroid surgery. Harmonic Scalpel, Harmonic Focus versus Conventional Hemostasis: A meta-analysis. Int J Surg 2016;28 Suppl 1:S22-32.

8. Cirocchi R, D'Ajello F, Trastulli S, et al. Meta-analysis of thyroidectomy with ultrasonic dissector versus conventional clamp and tie. World J Surg Oncol 2010;8:112.

9. Halsted WS, Evans HM. I. The Parathyroid Glandules. Their Blood Supply and their Preservation in Operation upon the Thyroid Gland. Ann Surg 1907;46:489-506.

10. Park I, Rhu J, Woo JW, et al. Preserving Parathyroid Gland Vasculature to Reduce Post-thyroidectomy Hypocalcemia. World J Surg 2016;40:1382-9.

11. Papavramidis TS, Anastasiou O, Pliakos I, et al. Parathyroid Function after Total Thyroidectomy: A Randomized Clinical Trial Concerning the Influence of the Surgical Technique. Endocr Pract 2018;24:150-5.

12. Haugen BR, Alexander EK, Bible KC, et al. 2015 American Thyroid Association Management Guidelines for Adult Patients with Thyroid Nodules and Differentiated Thyroid Cancer: The American Thyroid Association Guidelines Task Force on Thyroid Nodules and Differentiated Thyroid Cancer. Thyroid 2016;26:1-133.

13. American Thyroid Association Surgery Working Group; American Association of Endocrine Surgeons,; American Academy of Otolaryngology-Head and Neck Surgery, et al. Consensus statement on the terminology and classification of central neck dissection for thyroid cancer. Thyroid 2009;19:1153-8.

14. Olson JA Jr, DeBenedetti MK, Baumann DS, et al. Parathyroid autotransplantation during thyroidectomy. Results of long-term follow-up. Ann Surg 1996;223:472-8; discussion 478-80.

15. Selberherr A, Scheuba C, Riss P, et al. Postoperative hypoparathyroidism after thyroidectomy: efficient and cost-effective diagnosis and treatment. Surgery 2015;157:349-53.

16. Markogiannakis $\mathrm{H}$, Kekis PB, Memos N, et al. Thyroid surgery with the new harmonic scalpel: a prospective randomized study. Surgery 2011;149:411-5.

17. Papavramidis TS, Sapalidis K, Michalopoulos N, et al. UltraCision harmonic scalpel versus clamp-and-tie total thyroidectomy: a clinical trial. Head Neck 2010;32:723-7.

18. Miccoli P, Berti P, Dionigi G, et al. Randomized controlled trial of harmonic scalpel use during thyroidectomy. Arch Otolaryngol Head Neck Surg 2006;132:1069-73.

19. Voutilainen PE, Haglund CH. Ultrasonically activated shears in thyroidectomies: a randomized trial. Ann Surg 2000;231:322-8.

20. Ortega J, Sala C, Flor B, et al. Efficacy and costeffectiveness of the UltraCision harmonic scalpel in thyroid surgery: an analysis of 200 cases in a randomized trial. J Laparoendosc Adv Surg Tech A 2004;14:9-12.

21. Ondik MP, McGinn J, Ruggiero F, et al. Unintentional parathyroidectomy and hypoparathyroidism in secondary central compartment surgery for thyroid cancer. Head Neck 2010;32:462-6.

22. Chew $\mathrm{C}, \mathrm{Li} \mathrm{R}, \mathrm{Ng} \mathrm{MK}$, et al. Incidental parathyroidectomy during total thyroidectomy is not a direct cause of postoperative hypocalcaemia. ANZ J Surg 2018;88:158-61.

23. Lang BH, Chan DT, Chow FC. Visualizing fewer parathyroid glands may be associated with lower hypoparathyroidism following total thyroidectomy. Langenbecks Arch Surg 2016;401:231-8.

24. Chang YK, Lang BHH. To identify or not to identify parathyroid glands during total thyroidectomy. Gland Surg 2017;6:S20-9.

25. Cui Q, Li Z, Kong D, et al. A prospective cohort study of novel functional types of parathyroid glands in thyroidectomy: In situ preservation or autotransplantation? Medicine (Baltimore) 2016;95:e5810.

26. Zhu J, Tian W, Xu Z, et al. Expert consensus statement on parathyroid protection in thyroidectomy. Ann Transl Med 2015;3:230.

27. Lavazza $M$, Liu X, Wu C, et al. Indocyanine greenenhanced fluorescence for assessing parathyroid perfusion during thyroidectomy. Gland Surg 2016;5:512-21.

28. Li Y, Jian WH, Guo ZM, et al. A Meta-analysis of Carbon Nanoparticles for Identifying Lymph Nodes and Protecting Parathyroid Glands during Surgery. Otolaryngol Head Neck Surg 2015;152:1007-16.

Cite this article as: Jiang $\mathrm{J}$, Shen $\mathrm{M}, \mathrm{Lu} \mathrm{H}$. Ultrasonic scalpel with knot tying protects parathyroid function for total thyroidectomy with central neck dissection. Gland Surg 2020;9(2):192-199. doi: 10.21037/gs.2020.01.12 